\title{
CARACTERIZACIÓN DEL SISTEMA DE PRODUCCIÓN CAPRINO GRANJA EXPERIMENTAL DE LA UNIVERSIDAD FRANCISCO DE PAULA SANTANDER SEDE OCAÑA, COLOMBIA.
}

\author{
Characterization of the caprine production system experimental farm at the \\ University Francisco de Paula Santander Ocaña, Colombia.
}

\section{Artículo de Investigación}

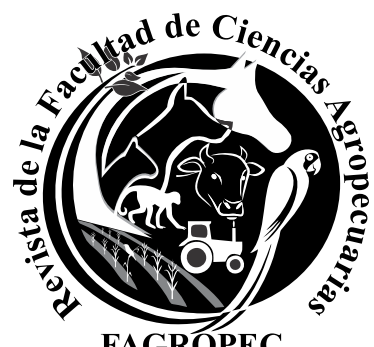

Recibido 15 de enero de 2020. Aceptado 3 de marzo de 2020.

${ }^{1}$ Docente tiempo completo, Universidad Francisco de Paula Santander, Grupo de Investigación GI@DS Y GIPAB. Sede el Algodonal, Ocaña, Colombia

https://orcid.org/0000-0002-0377-4664

${ }^{2}$ Fundación de Estudios Superiores COMFANORTE, Grupo de Investigación GRINFESC y GIPAB Cúcuta, Colombia

https://orcid.org/0000-0001-7718-853X

${ }^{3}$ Docente tiempo completo, Universidad Francisco de Paula Santander, Grupo de Investigación GI@DS Y GIPAB. Sede el Algodonal, Ocaña, Colombia

iD https://orcid.org/0000-0003-1971-8365

${ }^{4} \mathrm{Ph} . \mathrm{D}$. Ciencias agrarias

(iD https://orcid.org/0000-0002-1087-3150
Johann Fernando Hoyos Patiño ${ }^{1 *}$, Blanca Liliana Velazquez ${ }^{2}$, Daniel Antonio Hernández Villamizar ${ }^{3}$, Nancy Rodríguez Colorado ${ }^{4}$, Naudin Alejandro Hurtado Lugo

El estudio tuvo el propósito de caracterizar el sistema de producción caprino de la granja experimental de la Universidad Francisco de Paula Santander sede Ocaña (UFPSO), con el fin de reconocer los componentes del sistema productivo, tomando como punto diferenciador el hecho de ser un aprisco que presta servicios a la investigación y la academia. El tipo de investigación desarrollada fue cualitativo de tipo descriptivo, con muestreo no probabilística por conveniencia. Se aplicó una encuesta como instrumento para la recolección de datos al Director del sistema, al Zootecnista encargado de la reproducción y al Médico Veterinario responsable de la sanidad. Dicha encuesta, comprendió ocho factores que abarcan los componentes agrícola, pecuario, económico y socio-cultural; estudiando parámetros como: generalidades del sistema de producción; manejo nutricional y alimentario; inventario animal y genética existente; proceso productivo y manejo reproductivo; manejo sanitario; componente económico; y mercadeo y proceso administrativo. El sistema es semiestabulado, dedicado a la producción especializada de leche y genética para tal fin. Se observó rigurosidad en el manejo de registros productivos, sanitarios y reproductivos, mostrando fortalezas en estrategias de innovación en los procesos de transformación de lácteos y cárnicos elaborados con los machos descartados. Igualmente, se implementan procesos de medición del bienestar animal, estudios para la determinación de sostenibilidad de la producción y conservación de razas criollas, con procesos biotecnológicos en cabras de la raza Santandereana. El aprisco de la UFPSO, es referente para el desarrollo del sector caprino de la región, brindando capacitaciones, asesorías y genética a precios asequibles, impactando positivamente en la región. Por otro lado, presenta puntos a mejorar principalmente en la determinación de costos de producción, debido a las características de entidad pública que lo rige y los sobrecostos que esto conlleva.

Palabras claves:

Aprisco, semiestabulado y registros.

*Autor para Correspondencia:

jfhoyosp@ufpso.edu.co 
Como citar:

HOYOS PATIÑO, Johann Fernando; et al. Caracterización del sistema de producción caprino granja experimental de la Universidad Francisco de Paula Santander sede Ocaña, Colombia. En: Revista Facultad Ciencias Agropecuarias - FAGROPEC. Universidad de la Amazonia, Florencia - Caquetá. Volumen 12 enero-junio, 2020. Pp. 33-44 ISSN en Línea: 2539-178X.

https://doi.org/10.47847/fagropec.v12n1a3

\begin{abstract}
The purpose of this study is to characterize the goat production system of the experimental farm of the Universidad Francisco de Paula Santander (UFPSO) in Ocaña, in order to recognize the components of the production system, taking as a differentiating point, the fact that it is a sheepfold that provides services to research and academia. The type of applied research was qualitative of descriptive type, with non-probabilistic sampling for convenience. A survey was applied as an instrument for the collection of data to the Director of the system, the Zootechnician in charge of reproduction and the Veterinarian responsible for health. This survey includes 8 factors that cover the agricultural, livestock, economic and socio-cultural components, studying parameters such as: generalities of the production system; nutritional and food management; existing animal and genetic inventory; productive process and reproductive management; sanitary management; economic component; marketing and administrative process. The system is semi-stable, dedicated to the specialized production of milk and genetics for this purpose. Rigorous management of productive, sanitary and reproductive records was observed, showing strengths in innovation strategies in the transformation processes of dairy and meat products made from discarded males; these products are marketed at the University's point of sale and in the area's supermarkets. Likewise, processes for measuring animal welfare, studies to determine the sustainability of production and conservation of local breeds are implemented, with biotechnological processes in Santandereana goats. The UFPSO sheepfold is a reference for the development of the goat sector, providing training, advice and genetics at affordable prices, which has a positive impact on the region. On the other hand, it presents points to improve in the determination of production costs, due to the characteristics of public entity that governs it and the surcharges that this entails.
\end{abstract}

Key words:

Sheepfold, semi-stable and records.

\section{INTRODUCCIÓN}

El enfoque sistémico es una forma de analizar sistemas de producción, que requiere, para su valoración, la participación de múltiples disciplinas. Este tipo de evaluación permite explicar, conocer e intervenir aspectos complejos, como los procesos agropecuarios, permitiendo abordar más correctamente sus componentes e interacciones internas, con el medio ambiente (Marshall y Col., 1994; Escudero, 1998; Monteverde, 2013).

Los sistemas de producción agropecuarios se consideran como una forma de organización social productiva, con un nivel de desarrollo tecnológico adaptado a su ambiente, que busca la obtención de productos para subsistencia o comercialización (Rodrigues et al., 2003; González et al., 2014). Dada su naturaleza, presentan una alta complejidad, pero con el uso de procedimientos estructurados y lógicos se pueden caracterizar identificando sus fortalezas y debilidades. Se debe tener la capacidad para la aplicación de instrumentos que identifiquen sus componentes e interacciones. Spedding, 
1975 citado por Bárcenas et al. (2006), sugiere nueve parámetros para conceptualizar un sistema: propósito, límites, entorno, componentes (agrícola, pecuario, económico y socio cultural), interacciones, recursos, ingresos o insumos, egresos o salidas y subproductos. Este proceso, se puede ampliar caracterizando pilares productivos como: nutrición, manejo, sanidad, genética y bienestar animal.

La cabra (Capra aegagrus hircus), desde hace generaciones, es de gran utilidad y servicio para el hombre, destacándose por ser un animal de fácil adaptabilidad a las condiciones ambientales, sistemas de producción y diversos tipos de alimentación (Bolaños y Sanchez 1992 citado por Guerrero, et al., 2012). El auge por la producción de pequeños rumiantes en el País viene aumentando en los últimos años (Acero, 2014). El municipio de Ocaña, no es ajeno a estas tendencias, con un arraigo cultural por el consumo de todos los productos derivados de este tipo de explotaciones (PDMO, 2016). En la actualidad, existen campos aprisco y rediles en crecimiento, enfocados a la producción de leche con la explotación de razas especializadas como la Saanen, Alpino y Canario, y explotaciones ovinas que se valen de sistemas de cruzamiento de especímenes criollos con razas de gran habilidad materna, como la Katahdin, incursionan en los mercados cárnicos de la zona.

El municipio de Ocaña se ubica en la zona Centro Occidental del departamento y pertenece a la subregión noroccidental, limitando por el Oriente con los municipios de San Calixto, La Playa y Abrego. Al Norte con los municipios de Teorama, Convención y El Carmen. Al Sur con el municipio de Ábrego. Por el Occidente. Con los municipios de San Martín y Río de Oro (Alcaldía de Ocaña, 2018).

En el contexto Nacional, Ocaña hace parte del Norte de Santander ubicado sobre la cordillera Oriental en una zona completamente montañosa, tiene una extensión aproximada de $627.72 \mathrm{Km}^{2}$ que representa el 2,76 \% del área total del departamento, la cabecera municipal se encuentra a una distancia de $203 \mathrm{~km}$ de la capital del departamento por la vía Ocaña-Cúcuta, además se comunica con el departamento del Cesar en la vía Río de Oro-Aguachica, empalmando con la carretera que va a la Costa Atlántica y hacia el centro del país, de igual manera se comunica con la capital del departamento por la vía Convención - Tibú - Cúcuta (Alcaldía de Ocaña, 2018).

La Universidad Francisco de Paula Santander sede Ocaña (UFPSO) y específicamente de Decanatura de Ciencias Agrarias y del Ambiente, viene realizando grandes esfuerzos para el desarrollo de este tipo de sistemas en la región, promoviendo su expansión con los estudiantes del programa de Zootecnia.

El objetivo de esta caracterización, es describir y dar a conocer las potencialidades y puntos a mejorar de dicho sistema; sirviendo así como material de referencia para investigaciones futuras sobre el tema.

\section{MATERIALES Y MÉTODOS}

El método de investigación cualitativo (Bernal, 2016), de tipo descriptivo, ya que reseña las características de un fenómeno existente, a través de la recolección, interpretación y análisis de datos, en atención al universo real de donde proviene (Arias, 2006); para esto se realizará un 
muestreo no probabilístico por conveniencia (Bernal, 2016). El sistema de producción caprino de la UFPSO, se desarrolla en la Granja Experimental, ubicada a la orilla derecha del río Algodonal, con una extensión de 105 ha, 1150 m.s.n.m, promedio temperatura de $23^{\circ} \mathrm{C}$, humedad relativa del $70 \%$.

Esta caracterización hace parte del desarrollo del proyecto "Evaluación de la sostenibilidad utilizando indicadores de la metodología MESMIS en unidades productivas ovino-caprinas del municipio de Ocaña, Norte de Santander", el cual, utilizando indicadores determinados participativamente con los productores, valora el nivel de sostenibilidad de cuatro sistemas de producción de ovinos y caprinos, evaluando las dimensiones económica, ambiental y social.

Para la recolección de la información se utilizó una encuesta aplicada al Director del sistema, al Zootecnista encargado de la reproducción y al Médico Veterinario responsable de la sanidad. Dicho instrumento comprende ocho factores que abarcan los componentes agrícola, pecuario, económico y socio-cultural, estudiando parámetros como: generalidades del sistema de producción; manejo nutricional y alimentario; inventario animal y genética existente; proceso productivo y manejo reproductivo; manejo sanitario; componente económico; mercadeo y proceso administrativo.

\section{RESULTADOS Y DISCUSIÓN}

\section{Matriz Perfil de Capacidad Interna (PCI).}

De acuerdo al modelo de matriz Perfil de capacidad interna PCI, planteado por Téllez y Cubillos (2004), se identificaron, desde la óptica del investigador, las fortalezas y debilidades (FD) por medio de la evaluación de seis componentes (agrícola, pecuario, económico, sociocultural, proceso de mercadeo y gestión ambiental) y 46 indicadores en el sistema de producción caprinos de la Universidad Francisco de Paula Santander sede Ocaña (Tabla 1).

En el proyecto caprino de la UFPSO, el componente agrícola, se encuentra dentro de las fortalezas presentado pastoreo rotacional, y como punto a mejorar la falta de sistemas silvopastoril, el cual tiene como finalidad suministrar forraje a los animales y contribuir a mejorar la productividad, el bienestar animal y disminuir los efectos negativos ambientales (Navas, 2010).
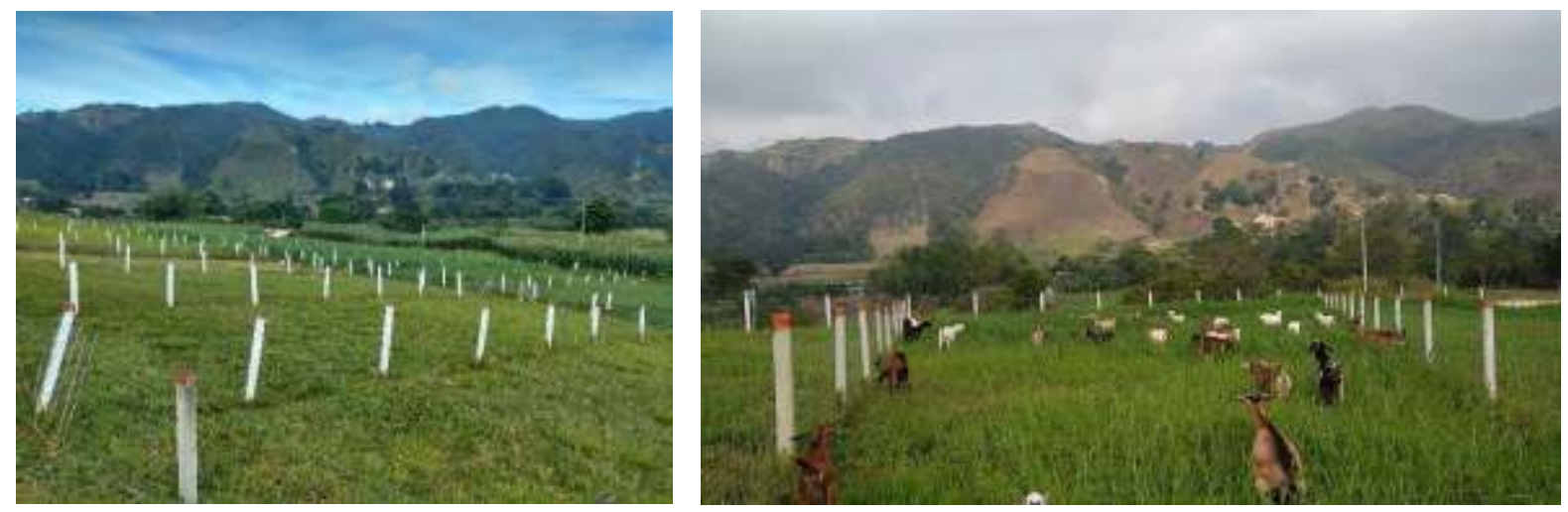

Figura 1. Rotación de potreros 
Tabla 1.

Matriz Perfil de Capacidad Interna (PCI) del proyecto caprino de la UFPSO.

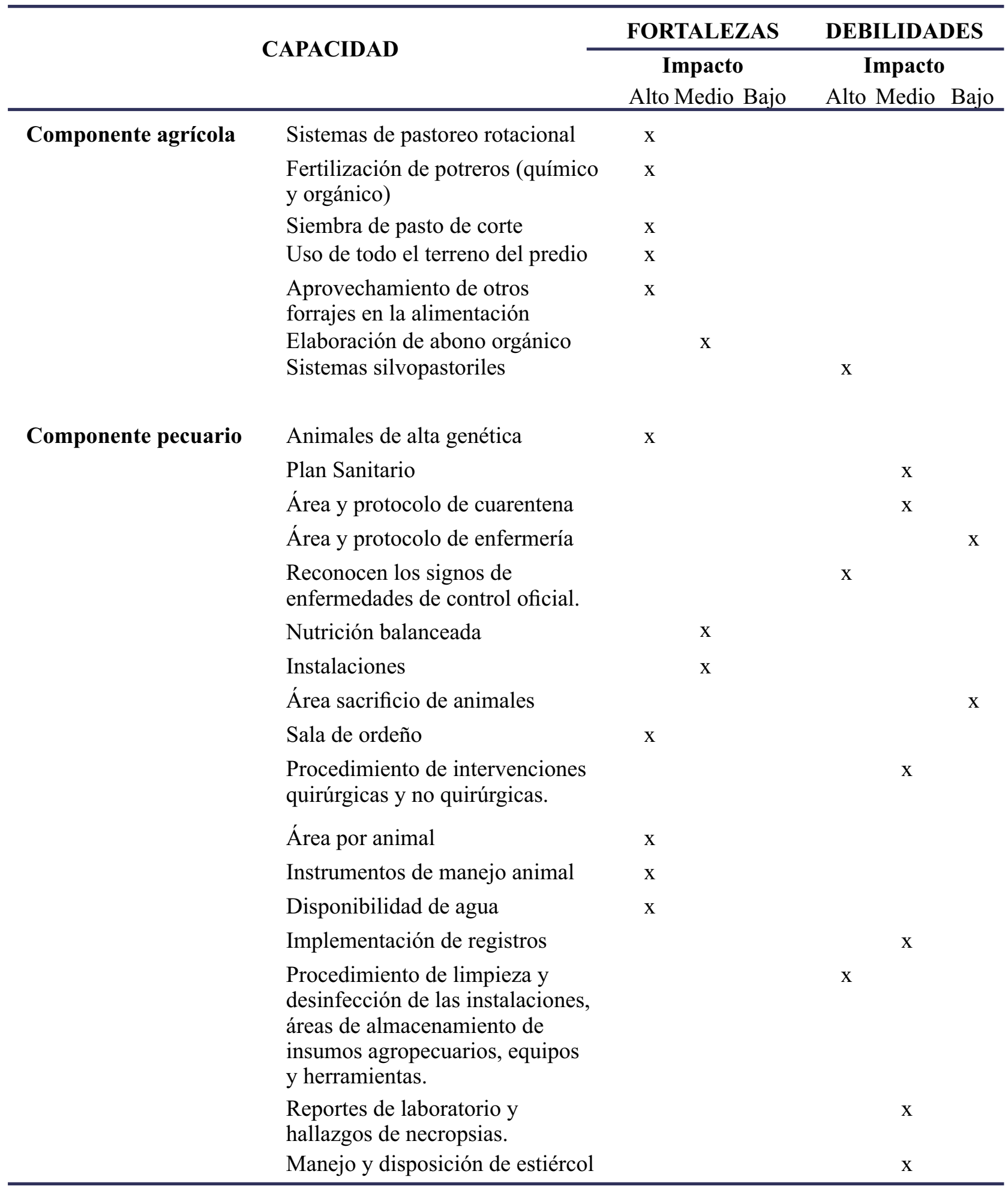


Continuación Tabla 1.

Matriz Perfil de Capacidad Interna (PCI) del proyecto caprino de la UFPSO.

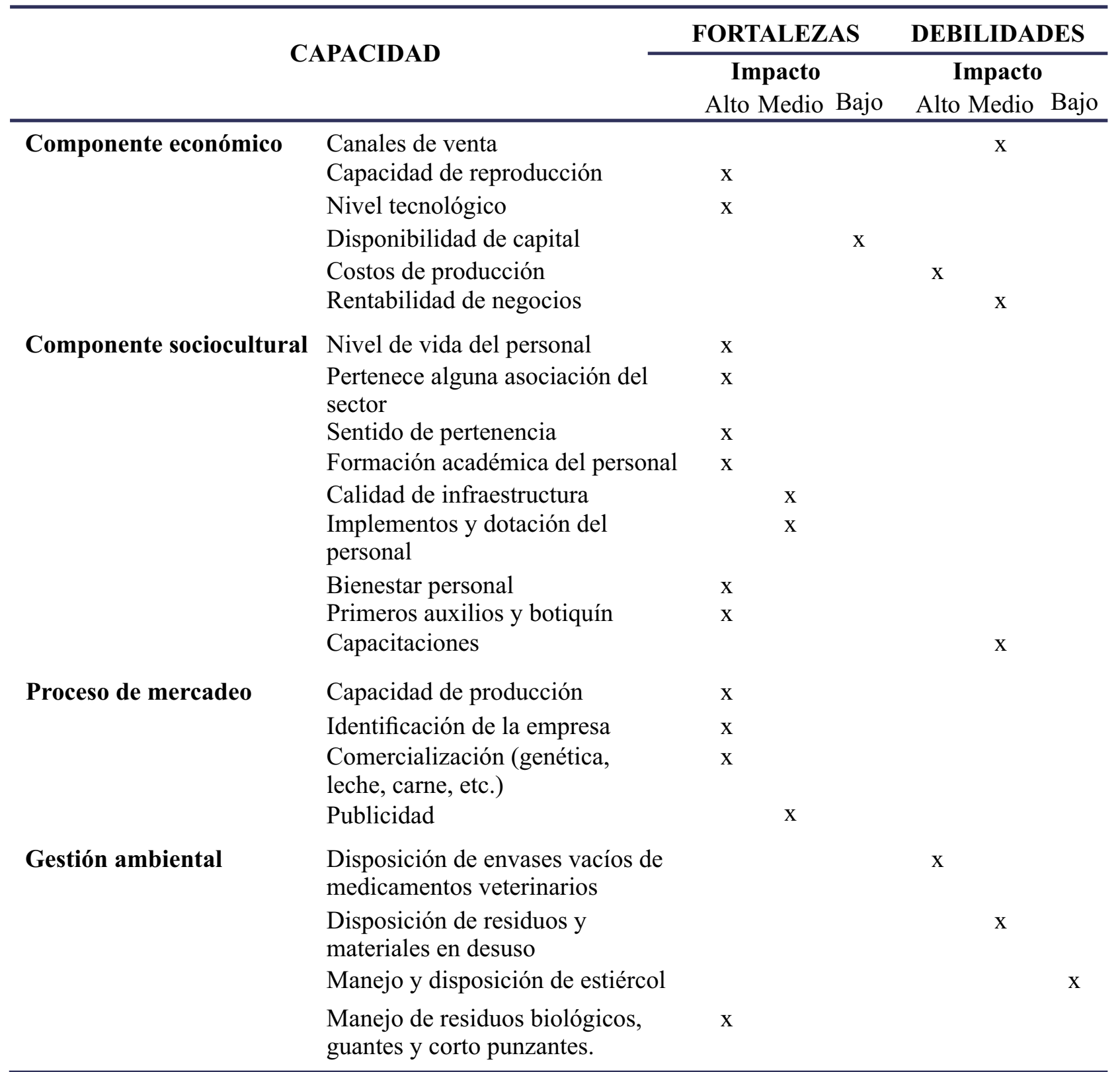

Nota: La tabla muestra la matriz de PCI con sus respectivas fortalezas y debilidades que presenta el proyecto caprino de la UFPSO.

En el componente pecuario, una de las fortalezas es la sala de ordeño que cuenta con un sistema de ordeño mecánico. Dentro de los puntos a mejorar está la elaboración del plan sanitario, potrero de cuarentena y enfermería; reconocimiento de los signos clínicos de las enfermedades de control oficial y la realización de los reportes de laboratorio y hallazgos de necropsias; elaboración de los procedimiento de limpieza y desinfección de las instalaciones, áreas de almacenamiento de insumos agropecuarios, equipos y herramientas; el manejo y disposición de estiércol y la implementación de 
registros para cumplimiento de la Resolución $\mathrm{N}^{\mathrm{o}} 2304$ de 2015 del Instituto Colombiano Agropecuario - ICA "por la cual se establecen los requisitos sanitarios y de inocuidad para obtener la certificación en buenas prácticas ganaderas bpg en la producción primaria de ovinos y caprinos destinados al sacrificio para consumo humano".
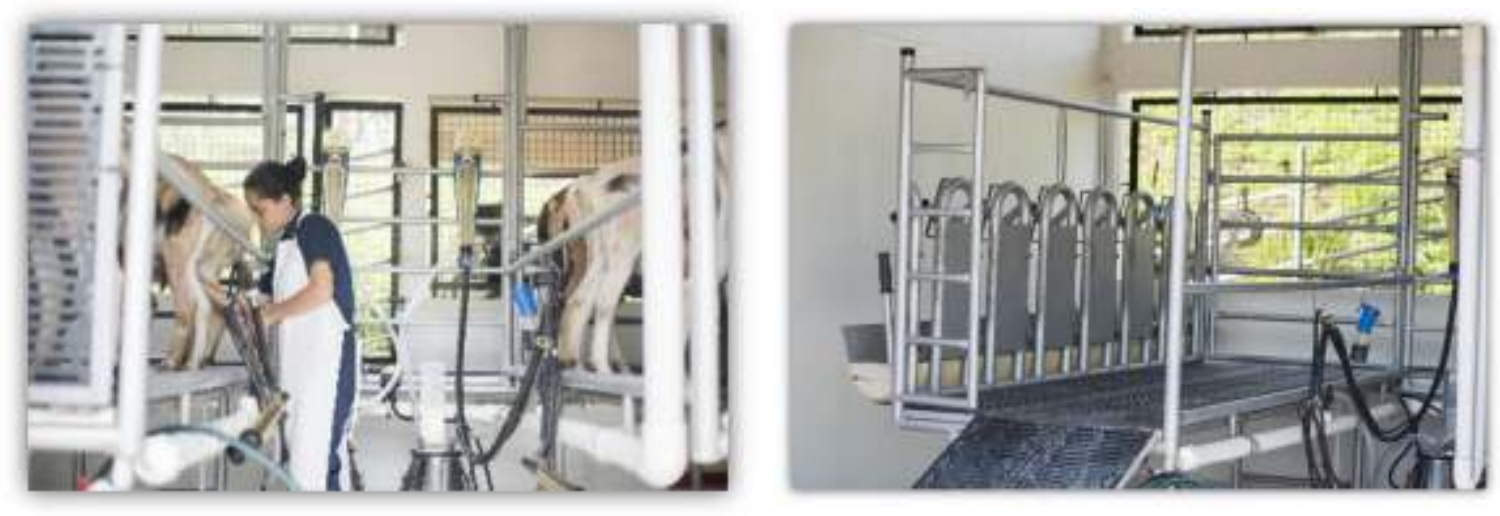

Sala de ordeño

Para el componente económico, una de las fortalezas es el uso de tecnologías, y dentro de los puntos a mejorar son los costos de producción, que son altos debido a que el propósito del proyecto caprino es la academia.

En el componente sociocultural, unas de las fortalezas encontradas fue la formación académica del personal y la participación como miembros a la Asociación Nacional de Capricultores y Ovinocultores (ANCO), y dentro de los puntos a mejorar se encuentra la necesidad de capacitación constante del personal encargado en temas relacionados con el manejo animal.

En los procesos de mercado, presenta fortalezas en capacidad de producción y comercialización de animales de alta genética, leche y carne de buena calidad. En gestión ambiental, una de las fortalezas fue el manejo adecuado de residuos biológicos, y dentro de los puntos a mejorar se encuentra la disposición final de los envases de medicamentos veterinarios y materiales en desuso.

A continuación, se presentan los datos obtenidos en la encuesta, que muestran las características del sistema de producción y reflejan la opinión y datos suministrados por los encuestados.

Generalidades del sistema de producción: el objetivo principal del sistema es la producción especializada de leche y genética caprina, aplicando un modelo de explotación semi-estabulada. Sumado a esto, presta apoyo al desarrollo académico e investigativo del programa de Zootecnia, dándole particularidades propias. El aprisco se georeferencia en las coordenadas $\mathrm{N} 08^{\circ} 14.485^{\prime} \mathrm{W}$ $073^{\circ} 19.081^{\prime}$ a 1215 m.s.n.m. (Granja Experimental UFPSO, 2019).

El sistema cuenta con 2,31 ha, distribuidas de la siguiente forma: área de pastoreo dividida en 27 lotes que suman un área de 0,94 ha, equivalente al 40,7\% del área total, sembradas con una combinación de Mombasa (ㅁaicum máximum) y Pará (Brachiaria mutica); zonas de pastoreo con 0.15 ha, que 
representa el 6,6\% del área total; banco forrajero con un área de 1,22 ha. equivalente al 52,7\%, sembradas en Botón de oro (Tithonia diversifolia), Alfalfa (Medicago sativa) y Sorgo Forrajero (Sorghum vulgare). Dichos cultivos, son abonados con la caprinaza producto del sistema, previo proceso de reposo de un mes.

Dispone con suministro de agua de riego del río Algodonal; el agua de bebida para los animales es tomada del acueducto Municipal y el agua para el lavado de instalaciones, es transportada por manguera de la quebrada las Lizcas. Cuenta con asistencia técnica permanente de tres profesionales: un Zootecnista encargado de la coordinación y administración, un Zootecnista para los procesos reproductivos, y un Médico Veterinario encargado de la sanidad. Como personal de apoyo cuenta con un operario a tiempo completo, un pasante permanente del programa de Zootecnia y dos becas trabajo durante el periodo académico.

En infraestructura para alojamiento y manejo animal, el sistema de producción está dotado con un aprisco elevado de $120 \mathrm{~m}^{2}$ con capacidad para 72 animales adultos, pisos en estiva plástica, techo en zinc; un segundo aprisco en suelo de $135 \mathrm{~m}^{2}$, techo zinc, separada por rejas metálicas, con capacidad de 60 animales adultos; los bebederos son automáticos, saladeros en PVC; corral de manejo área 72 $\mathrm{m}^{2}$ con manga central; sala de ordeño mecánico, de tipo tandem Delaval de dos puestos con capacidad para cuatro puestos; bascula electrónica y cobertizo para el forraje. En infraestructura administrativa, cuenta con oficina, baño, laboratorio reproductivo, depósitos para concentrado y herramienta.
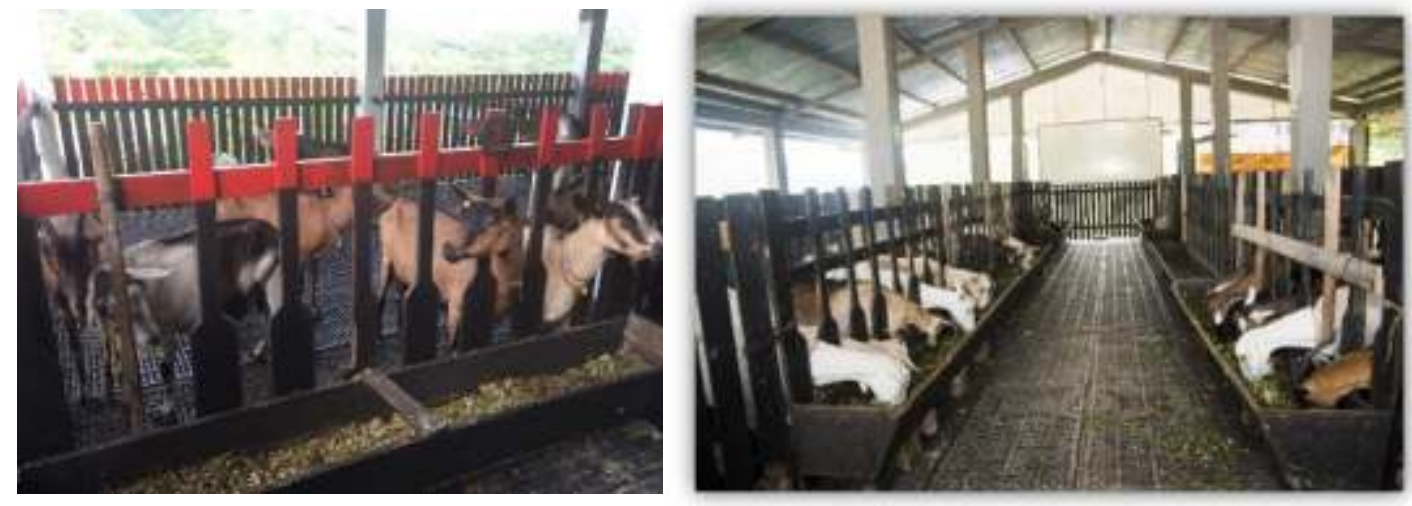

Apriscos y comederos

Manejo nutricional y alimentario: se desarrolla un sistema rotacional de potreros donde los animales pastorean de 8:30 a 11:30 a.m., el resto del día permanecen en los apriscos. A los cabritos se les suministra un litro leche diario, en tres tomas hasta el mes y después en dos tomas hasta cumplir 3 meses de edad. A las cabretonas se les complementa la dieta con 300 gr de concentrado día. A las cabras en producción se les suministra 500 gr de concentrado comercial día, con un contenido de PC del 16\%; en el sistema de producción se eleva el porcentaje de concentrado del 20\% PC. Permanentemente, se suministra sal mineralizada comercial al $8 \%$ de fosforo.

Inventario animal y genética existente: los datos son los reportados a octubre del año 2018 con un total de caprinos de 96, divididos de la siguiente forma: hembras 82, machos 12 y crías 2, de las razas Saanen, Alpina, Toggenburg, Canaria, Santandereana, Nubiana y sus cruces. Los machos se descartan por no cumplir con parámetros de peso y morfología, todos los machos media sangre se 
esterilizan quirúrgicamente, aproximadamente a los dos meses edad, mediante el método anillo de goma o liga, y algunos a testículo abierto como practica académica. Son cebados y se transforman en productos cárnicos en la planta de proceso agroindustriales de la Universidad. Los machos seleccionados para cría son vendidos a precio asequibles como programa de fomento del sistema de producción en la zona.

Proceso productivo y manejo reproductivo: se estipulan en el sistema de producción los siguientes rangos de edad para determinar el momento productivo de los animales: lactantes hasta el 1 mes de nacidos, levante hasta 4 meses, 12 meses cría y producción. La edad al primer servicio es 16 a 20 meses, el primer parto 21 a 25 meses. El porcentaje de fertilidad no es alto, debido a los aspectos académicos, donde los estudiantes en ciertos lotes de hembras, realizan el proceso de inseminación y esto disminuye la efectividad de este parámetro; el porcentaje de nacimientos es del 90\%; se presentan casos aislados de reabsorciones.

Según los datos promedio en registros, el peso promedio al nacimiento es $2 \mathrm{~kg}$, para el destete es de 8 $\mathrm{Kg}$ peso con 30 días edad. Para el primer servicio el peso promedio es peso 35 a $40 \mathrm{~kg}$ y 16 meses edad. Para los machos dependiendo el desarrollo se consideran los 12 meses para iniciar el proceso de monta. En la actualidad el promedio de producción de leche es (1) litro día, con una lactancia de 5 meses. Los machos y hembras se descartan por producción o por vejez; todas las hembras nacidas se conservan para reemplazo y aumentar el pie de cría.

Dentro del proceso reproductivo, rara vez se usa la monta directa, predominan las biotecnologías como la inseminación transcervical en lotes de 8 hembras e inseminación laparoscópica, en lotes de 15 hembras. Se está incursionando con gran éxito en la transferencia de embriones, fortaleciendo la conservación del germoplasma de la raza Criolla Santandereana. Se sincroniza con esponja vaginal y se recela diariamente con el macho. La verificación del estado de preñez se realiza con ultrasonido. Se tiene destinado un corral de maternidad y las hembras se ingresan por fecha de posible parto, curando el ombligo de las crías con yodo. Esporádicamente, se presentan abortos, los registros muestran que las principales causas se deben a golpes. El porcentaje de partos gemelares es del $50 \%$.

Dentro de los procesos rutinarios del aprisco se encuentran los arreglos pódales en los meses de febrero y agosto (motivos académicos), antes de las ferias y cuando los animales lo requieran.

Destinado a la academia e investigación, existe un lote receptoras, donde se realizan ensayos de nutrición y suplementación. Igualmente, prácticas de inseminación.

Manejo sanitario: el sistema de producción cuenta con un Médico Veterinario constante, el cual lleva el registro de todos los eventos sanitarios del aprisco (vacunaciones, desparasitaciones y tratamientos). Se realiza control mensual de Haemonchus contortus, con el uso del sistema FAMACHA y coprológicos a los animales que presenten sintomatologías. El médico Veterinario cumple funciones académicas y acompaña las prácticas de inseminación y aplicación de hormonales en el plantel.

Mercadeo: los productos como leche y carne son procesados en el laboratorio de cárnicos y lácteos, teniendo ya estandarizados las formulaciones de quesos, dulces, helados, chorizos y salchichas. 
Estos se comercializan en la región, aprovechando el punto de venta que la Universidad tiene, para el expendio de los productos de la granja experimental.

Por otro lado, se comercializa leche fresca en promedio a 5.000 COP litro y se hace fomento y extensión a la comunidad con la venta de cabretones de buena genética lechera, con pesos que oscilan entre 20 a 25 kilos y precio entre 120.000 a 200.000 COP.

Proceso administrativo: en la explotación caprina de la UFPSO, se implementan estrategias de planeación, organización, dirección y control, con el fin de tener los objetivos y metas claras; se implementan registros productivos y sanitarios, llevando control de inventario y reproducción del plantel, mediante el software OVISWEB. El sistema de identificación de animales se hace mediante el tatuado y chapeteado al mes de nacidos.

El aprisco de la UFPSO, ha realizado participaciones destacada en los últimos 7 años en ferias de renombre nacional, como: del 2012-2018 en CENFER y Valledupar 2018, con resultados destacados obteniendo títulos de grandes capeones en las razas Alpino, Saanem y Santandereano; Campeón supremo con ejemplares de la raza Alpino y mejores criadores y expositores de la feria.

Todo el proceso de compras en el aprisco, se realiza bajo la figura de fondo rotatorio con el que cuenta la granja experimental; anualmente se proyecta la inversión y costos de mantenimiento para su posterior ejecución.

\section{CONCLUSIONES}

El proceso productivo presente en el aprisco de la Universidad Francisco de Paula Santander sede Ocaña, sirve como punto de referencia para el desarrollo del sector caprino en la región, prestando servicios de asesoría y extensión para el mejoramiento del pequeño productor.

El aporte académico de este sistema de producción, a la formación del Zootecnista es incalculable, brindando los espacios para la integración del componente teórico con el práctico, en cada fase del proceso productivo, haciendo participe al estudiante en la nutrición, planes sanitarios, mejoramiento genético, cría, manejo y presentación en ferias de los productos obtenidos del proceso de inseminación artificial y transferencia de embriones.

\section{LITERATURA CITADA}

ACERO, P. Victor, M. El bienestar animal en sistemas productivos de ovinos-caprinos en Colombia. En: Spei Domus, 10(21).Bogota: Colombia. 2014. Pp 57-62. \{En línea\}.\{13 Octubre de 2019\} Disponible en: https://bit.ly/2klpPyH

ALCALDIA DE OCAÑA. Sitio oficial alcaldía de ocaña. Norte de Santander. 2018. \{En línea\}.\{11 de Noviembre de 2019\} Disponible en: http:/www.ocana-nortedesantander.gov.co/menu/

ARIAS. El proyecto de investigación introducción a la metodología científica. 5ta edición. Episteme. 2006.

BÁRCENAS, Iveth D; AGUIRRE, Leosber M y GARCÍA, Yaderson W. Evaluación técnica y económica de 
tres sistemas productivos pecuarios en el municipio de león en el período de octubre del 2005 a marzo del 2006. En: Universidad Nacional autónoma de Nicaragua. UNAN - león. Facultad de ciencias. Departamento de ingeniería en agroecología tropical. 2006. \{En línea\}.\{20 Enero de 2019\} Disponible en: https://bit.ly/2xoJ80r.

BERNAL, Cesar, A. Metodología de la investigación. Administración, economía, humanidades y ciencias sociales. 3 Edición. Bogotá: Pearson, 2016. Pp 1-50. \{En línea\}.\{16 Enero de 2019\} Disponible en: https://cutt.ly/UyxNXKr

BOLAÑOS, B. Oscar y SÁNCHEZ,D. Olman. Elementos básicos para el manejo de animales de granja Cabras. En: UNED. San José: Costa Rica. 1992.

GONZÁLEZ, C., MANRIQUE, C., \& GRAJALES, H. Formulación de un modelo conceptual para la gestión de la información en la producción de ovinos y caprinos: 1. análisis de los sistemas de gestión y definición de las fronteras del modelo. En: Revista de la Facultad de Medicina Veterinaria y de Zootecnia, 61(3), 2014. Pp 284-301. \{En línea\}.\{17 Octubre de 2019\} Disponible en:https://bit.ly/2WMmJVt

GRANJA EXPERIMENTAL UFPSO. Proyectos en desarrollo en la granja experimental. Proyecto Caprino. En: Universidad Francisco de Paula Santander Ocaña. 2019. \{En línea $\}.\{21$ de febrero de 2019\} Disponible en:https://ufpso.edu.co/granja/Actividades

GUERRERO L.M; TELLEZ J.N; VERAM; GARZAB.A; RINCÓN J.L; MARTÍNEZ B.D; SERRANO N.C; VARGAS BAYONA, J.E. Caracterizacion de los sistemas de producción caprinos presentes en el Municipio de Jordan, Santander, Colombia. En: Actas Iberoamericanas de Conservación Animal AICA 2. 2012. Pp 171174. \{En línea\}.\{21 de Octubre de 2019$\}$ Disponible en: https://bit.ly/3ai1bDX

INSTITUTO COLOMBIANO AGROPECUARIO (ICA). Resolución N 2304 Por la cual se establecen los requisitos sanitarios y de inocuidad para obtener la certificación en Buenas Practicas Ganaderas BPG en la producción primaria de ovinos y caprinos destinados al sacrificio para consumo humano. 2015. \{En línea $\}.\{12$ de Octubre de 2019\} Disponible en: https://bit.ly/2Zs4ylu

MARSHALL, E.; BONNEVIALE, J.R.; FRANCFORT, I. Functionnement et diagnostic global de l'explotation agricole. ENESAD-SED. Dijon, Francia. 1994. P 173.

MONTEVERDE, S. Agroecología. El agroecosistema y conceptos del enfoque sistémico. En: Universidad de la República. Montevideo, Uruguay. 2013. \{En línea $\}.\{12$ de Noviembre de 2019\} Disponible en: https://bit.ly/39mYuQe

NAVAS, P. Alexander. Importancia de los sistemas silvopastoriles en la reducción del estrés calórico en sistemas de producción ganadera tropical. En: Revista de Medicina Veterinaria, (19). 2010. Pp 113-122. \{En línea $\}.\{13$ de Octubre de 2019\} Disponible en: https://bit.ly/2VhrnYu

PDMO. Plan de Desarrollo Municipio de Ocaña 2016-2019. "ES LA HORA DE OCAÑA". Municipio de Ocaña. 2016. \{En línea\}.\{13 de Febrero de 2019\} Disponible en: https://bit.ly/39js2ht

RECA, Lucio y ECHEVERRIA, Ruben. La visión y misión de la agricultura en el año 2020: hacia un enfoque que valorice la agricultura y el medio rural. Agricultura, medio ambiente y pobreza rural en América Latina. IFPRI-BID, Washington. 1998. Pp 21-54. \{En línea\}.\{13 Enero de 2019\} Disponible en: https://cutt.ly/wyxMvWT 
RODRIGUES, A.; TOMMASINO, H.; FOLADORI, G.; GREGORCZUC A. ¿Es correcto pensar la sustentabilidad a nivel local? Un análisis metodológico a partir del estudio de caso en un área de protección ambiental en el litoral sur de Brasil. Revista Theomai, $\mathrm{N}^{\mathrm{o}}$ 7.2003.

SPEDDING, C. R. W. The Biology of Agricultural Systems. Chapter 1, The Purposes of Agriculture. Academic Press, London. 1975. Pp 1-13

TÉLLEZ, G; CUBILLOS, A. Planeaciones estratégicas de empresas ganaderas. Capacitación en gestión para empresarios ganaderos. Módulo 4. En: Fedegan. Bogotá. 2004. \{En línea\}.\{16 de Diciembre de 2019$\}$ Disponible en: https://bit.ly/2Iu1AqX 